\title{
Effect of Thiol-reducing Agents and Antioxidants on Sulfasalazine-induced Hepatic Injury in Normotermic Recirculating Isolated Perfused Rat Liver
}

\author{
Reza Heidari ${ }^{1}$, Neda Esmailie ${ }^{2}$, Negar Azarpira ${ }^{3}$, Asma Najibi ${ }^{1,2}$ and Hossein Niknahad ${ }^{1,2}$ \\ 'Pharmaceutical Sciences Research Center, Shiraz University of Medical Sciences, Shiraz, Iran \\ ${ }^{2}$ Department of Pharmacology and Toxicology, Faculty of Pharmacy, Shiraz University of Medical Sciences, Shiraz, Iran \\ ${ }^{3}$ Transplant Research Center, Shiraz University of Medical Sciences, Shiraz, Iran
}

(Received April 10, 2015; Revised June 16, 2015; Accepted July 3, 2015)

\begin{abstract}
Sulfasalzine is a widely administered drug against inflammatory-based disorders in human. However several cases of liver injury are associated with its administration. There is no stabilized safe protective agent against sulfasalazine-induced liver injury. Current investigation was designed to evaluate if $\mathrm{N}$-acetylcysteine (NAC) and dithioteritol (DTT) as thiol reducing agents and/or vitamins C and E as antioxidants have any protective effects against sulfasalazine-induced hepatic injury in an ex vivo model of isolated rat liver. Rat liver was canulated and perfused via portal vein in a closed recirculating system. Different concentrations of sulfasalazine and/or thiol reductants and antioxidants were administered and markers of organ injury were monitored at different time intervals. It was found that $5 \mathrm{mM}$ of sulfasalazine caused marked liver injury as judged by rise in liver perfusate level of alanine aminotransferase (ALT), aspartate aminotransferase (AST), and lactate dehydrogenase (LDH) $(p<0.05)$. A significant amount of lipid peroxidation and hepatic glutathione depletion were detected in drug-treated livers, accompanied with significant histopathological changes of the organ. Administration of NAC $(500 \mu \mathrm{M})$, DTT $(400 \mu \mathrm{M})$, Vitamin C $(200 \mu \mathrm{M})$, or vitamin E $(200 \mu \mathrm{M})$ significantly alleviated sulfasalazine-induced hepatic injury in isolated perfused rat liver. The data obtained from current investigation indicate potential therapeutic properties of thiol reductants and antioxidants against sulfasalazine-induced liver injury.
\end{abstract}

Key words: Dithioteritol, Drug-induced liver injury (DILI), Glutathione, Hepatoprotection, N-acetyl cysteine, Oxidative stress

\section{INTRODUCTION}

Drug-induced liver injury (DILI) is a major clinical complication developed after administration of many pharmaceuticals (1-4). Sulfasalazine is widely used in inflammatory bowel disease and rheumatoid arthritis (5). However, hepatic injury due to sulfasalazine administration remains a clinical challenge. Several cases of sulfasalazine-induced liver injury are reported $(6,7)$, which might even be fatal $(8,9)$. The frequency of serious hepatic events associated with sul-

Correspondence to: Hossein Niknahad, Department of Pharmacology and Toxicology, Shiraz University of Medical Sciences, Shiraz, Iran

E-mail: niknahadh@sums.ac.ir

Abbreviations: DILI, Drug-induced liver injury; SSZ, Sulfasalazine; ROS, Reactive oxygen species.

This is an Open-Access article distributed under the terms of the Creative Commons Attribution Non-Commercial License (http:// creativecommons.org/licenses/by-nc/3.0) which permits unrestricted non-commercial use, distribution, and reproduction in any medium, provided the original work is properly cited. fasalazine administration is estimated at $0.4 \%$ of treated patients $(10,11)$. The precise mechanism(s) of hepatic injury induced by sulfasalazine is not fully understood. Moreover, there is no effective protective agent against this complication in human.

Some investigations proposed the role of oxidative stress and its consequent events in sulfasalazine-induced liver injury $(12,13)$. Impairment of cellular defense mechanisms against oxidative stress is also reported after sulfasalazine administration to animals $(12,14)$. Hence, it seems reasonable that antioxidants and thiol reducing agents such as Nacetyl cysteine (NAC) could reverse and/or alleviate liver injury induced by sulfasalazine.

Isolated organs are useful models for examining xenobiotics-induced injury without the complication of many interacting factors which are difficult to control in other experimental models $(15,16)$. Current study was designed to investigate the role of oxidative stress, in sulfasalazineinduced hepatic injury and finding a hepatoprotective agent with therapeutic potential against this complication. Different antioxidants and thiol-reducing agents were adminis- 
tered to evaluate if they are able to alleviate sulfasalazineinduced liver damage. Different concentrations of drug and hepatoprotective agents were perfused to liver and the biomarkers of hepatic injury including AST, ALT, and LDH were monitored in liver effluent buffer. Hepatic glutathione content and the amount of lipid peroxidation were assessed as markers of oxidative stress-induced tissue injury. Moreover, histopathological changes of the organ was evaluated at the end of each experiments.

\section{MATERIAL AND METHODS}

Chemicals. N-acetyl cysteine (NAC), and Dithioteritol were obtained from Acros (New Jersey, USA). Trichloroacetic acid (TCA), Ethylenediaminetetraacetic acid (EDTA), Vitamin E, and Vitamin C were obtained from Merck (Darmstadt, Germany). Kits for evaluating biomarkers of liver injury were obtained from Pars Azmun (Tehran, Iran). Thiobarbituric acid (TBA) was obtained from SERVA (Heidenberg, New York). All salts used for preparing buffer solutions were of analytical grade and obtained from Merck (Darmstadt, Germany).

Isolated perfused rat liver preparation. Male SpragueDawley rats (200 300 g) were purchased from Laboratory Animal Breeding Center, Shiraz University of Medical Sciences and allowed free access to food and tap water. The animals were handled and used according to the animal handling protocol approved by the ethics committee of Faculty of Pharmacy, Shiraz University of Medical Sciences, Shiraz, Iran (Reg. no. 93-01-36-7612). After anesthetizing the animals with pentobarbital $(50 \mathrm{mg} / \mathrm{kg}$, i.p.), the livers were canulated and perfused via portal vein $(16,17)$ with hemoglobin- and albumin-free Krebs-Henseleit buffer $\left(\mathrm{pH}=7.4,37^{\circ} \mathrm{C}\right)$ gassed with carbogen $\left(95 \% \mathrm{O}_{2}, 5 \% \mathrm{CO}_{2}\right)$. The perfusate was pumped through the livers with a peristaltic pump at a constant flow rate of $3 \mathrm{~mL} / \mathrm{min} / \mathrm{g}$ liver weight, in a re-circulating mode. The perfusate buffer volume was $200 \mathrm{~mL}$ in all experiments.

Study procedure. Isolated rat liver was exposed to different concentrations of sulfasalazine and other postulated hepatoprotective agents for 180 mins of perfusion. Hepatic injury was determined at scheduled time intervals during the experiment to evaluate the effects of various concentration of sulfasalazine on liver. The injurious concentration of the drug is reported as a concentration value, which lead to a significant rise in all assessed biomarkers of liver injury after 120 mins of organ perfusion. Samples were taken from liver perfusate at different times and assessed for biomarkers of liver injury. At the end of each experiment (180 mins), liver samples were used for lipid peroxidation, glutathione content and histopathological tests. Spectrophotometric scanning of the perfusate solutions revealed no physiochemical interaction between sulfasalazine and other protective agents in current system. Thiol reducing agents and antioxidants caused no sign of liver injury when they were administered alone at the mentioned concentrations.

Perfusate biochemistry. Mindray Random Access BS$200^{\circledR}$ auto analyzer (Mindray, Shenzhen, China) and Pars Azmun $^{\circledR}$ standard kits (Pars Azmun, Tehran, Iran) were used to measure ALT, AST, and LDH activities in liver perfusate at different time points (18).

Lipid peroxidation in liver tissue. Level of lipid peroxidation was assessed by measuring thiobarbituric acid reactive substances (TBARS) in different experimental groups. Briefly, reaction mixture consists of trichloroacetic acid $(15 \%$, $\mathrm{w} / \mathrm{v})$, thiobarbituric acid $(0.375 \%, \mathrm{w} / \mathrm{v}), \mathrm{HCl}(0.25 \mathrm{~N})$, and $0.2 \mathrm{~mL}$ of tissue homogenate. The mixture was shaked and heated in boiling water $\left(100^{\circ} \mathrm{C}\right)$ for $45 \mathrm{mins}$. When the reaction mixture was cooled, $2 \mathrm{~mL}$ of n-butanol was added and vigorously shaked. Samples were centrifuged $(3000 \mathrm{~g}$ for 5 mins) and the absorbance of developed color in n-butanol phase was read at $532 \mathrm{~nm}$ using an Ultrospec $2000^{\circledR}$ UV spectrophotometer (Pharmacia Biotech, Uppsala, Sweden) (19).

Hepatic glutathione content. The liver GSH levels were assessed by determining non-protein sulphydryl contents with the Ellman reagent (20,21). Briefly, samples of liver $(200 \mathrm{mg})$ were homogenized in ice-cooled EDTA $(20 \mathrm{mM})$. Five milliliter of liver homogenate was mixed with $4 \mathrm{~mL}$ of distilled water and $1 \mathrm{~mL}$ of trichloroacetic acid $(50 \% \mathrm{w} / \mathrm{v})$. The mixture was centrifuged $(10000 \mathrm{~g}$, $4^{\circ} \mathrm{C}$, for $25 \mathrm{mins}$ ). Two $\mathrm{mL}$ of the supernatant was mixed with $4 \mathrm{~mL}$ of Tris buffer $(\mathrm{pH}=8.9)$, and $100 \mu \mathrm{L}$ of DTNB $(0.01 \mathrm{M}$ in methanol) was added to the samples. The absorbance of developed color was measured in $412 \mathrm{~nm}$ using an Ultrospec $2000^{\circledR}$ UV spectrophotometer.

Liver histopathological evaluation. Samples of liver were fixed in neutral buffered formalin solution $(0.4 \%$ sodium phosphate monobasic, $\mathrm{NaH}_{2} \mathrm{PO}_{4}, 0.64 \%$ sodium phosphate dibasic, $\mathrm{Na}_{2} \mathrm{HPO}_{4}$, and $10 \%$ formaldehyde in distilled water). Paraffin-embedded sections of liver were prepared and stained with haematoxylin and eosin (H\&E) before light microscope viewing.

Statistical analysis. All data are expressed as the Mean \pm SEM. Comparison of data sets were performed by the one way analysis of variance (ANOVA) with Tukey's as the post hoc test. Differences were considered significant when $p<0.05$.

\section{RESULTS}

Isolated rat liver was exposed to different concentrations 
Table 1. Concentration-response of the isolated rat liver to sulfasalazine

\begin{tabular}{lccc}
\hline \hline Treatment & $\begin{array}{c}\text { Perfusate } \\
\text { ALT (U/L) }\end{array}$ & $\begin{array}{c}\text { Perfusate } \\
\text { AST (U/L) }\end{array}$ & $\begin{array}{c}\text { Perfusate } \\
\text { LDH (U/L) }\end{array}$ \\
\hline Control (only buffer) & $21 \pm 14$ & $34 \pm 4$ & $26 \pm 4$ \\
Sulfasalazine $125 \mu \mathrm{M}$ & $31 \pm 13$ & $44 \pm 9$ & $38 \pm 11$ \\
Sulfasalazine $250 \mu \mathrm{M}$ & $39 \pm 8$ & $137 \pm 53^{*}$ & $89 \pm 25$ \\
Sulfasalazine $500 \mu \mathrm{M}$ & $46 \pm 13$ & $113 \pm 4^{*}$ & $199 \pm 72^{*}$ \\
Sulfasalazine $1 \mathrm{mM}$ & $75 \pm 10$ & $159 \pm 52^{*}$ & $233 \pm 29^{*}$ \\
Sulfasalazine 5 mM & $601 \pm 148^{*}$ & $306 \pm 39^{*}$ & $355 \pm 143^{*}$
\end{tabular}

Data are shown as Mean \pm SEM for at least three separate experiments which were assessed after 120 mins of liver perfusion.

"Indicates significant increase as compared with control (Only buffer) group $(p<0.05)$.

of sulfasalazine (Table 1). It was found that $5 \mathrm{mM}$ of sulfasalazine caused a significant elevation of AST, ALT, and LDH in the perfusate after 120 mins of liver perfusion $(p<0.05)$ (Table 1$)$. The $5 \mathrm{mM}$ concentration of sulfasala- zine was considered as an injurious dose of the drug in this system and was applied for further investigation on sulfasalazine-induced liver injury.

The biomarkers of liver injury were assessed in different experimental groups after administrating thiol-reducing agents (NAC and DTT) and antioxidants (Vitamins C and E). It was found that NAC in both concentrations of $300 \mu \mathrm{M}$ and $500 \mu \mathrm{M}$ significantly decreased elevation of ALT (Table 2), AST (Table 3) and LDH (Table 4) in perfusate $(p<0.05)$. Administration of DTT as another thiol-reducing agent mitigated elevation in perfusate biomarkers of liver injury at the concentration of $400 \mu \mathrm{M}$ (Tables 2-4) $(p<0.05)$. The levels of ALT, AST and LDH were significantly lower in liver effluent as compared with sulfasalazine-treated group, when antioxidants such as Vitamin $\mathrm{C}$ $(200 \mu \mathrm{M})$ and $\mathrm{E}(200 \mu \mathrm{M})$ were administered $(p<0.05)$ (Tables 2-4).

Lipid peroxidation and hepatic glutathione content were assessed in different groups at the end of each experiment.

Table 2. Effect of thiol-reductants and antioxidants on ALT release from liver induced by sulfasalazine

\begin{tabular}{|c|c|c|c|}
\hline \multirow[b]{2}{*}{ Ttime (min): } & \multicolumn{3}{|c|}{ Perfusate ALT level (U/L) } \\
\hline & 60 & 120 & 180 \\
\hline Control (only buffer) & $13 \pm 2$ & $20 \pm 4$ & $21 \pm 8$ \\
\hline+ Sulfasalazine $5 \mathrm{mM}$ & $17 \pm 5$ & $677 \pm 113^{*}$ & $1811 \pm 107^{*}$ \\
\hline + NAC $300 \mu \mathrm{M}$ & $52 \pm 14$ & $122 \pm 23^{\mathrm{a}}$ & $288 \pm 140^{\mathrm{a}}$ \\
\hline+ NAC $500 \mu \mathrm{M}$ & $6 \pm 3$ & $33 \pm 7^{\mathrm{a}}$ & $120 \pm 35^{\mathrm{a}}$ \\
\hline + DTT $200 \mu \mathrm{M}$ & $4 \pm 1$ & $76 \pm 36$ & $244 \pm 27$ \\
\hline + DTT $400 \mu \mathrm{M}$ & $4 \pm 1^{\mathrm{a}}$ & $23 \pm 4^{\mathrm{a}}$ & $152 \pm 81^{\mathrm{a}}$ \\
\hline+ Vitamin E $200 \mu \mathrm{M}$ & $1.66 \pm 0.66^{\mathrm{a}}$ & $18 \pm 6^{\mathrm{a}}$ & $45 \pm 20^{\mathrm{a}}$ \\
\hline+ Vitamin C $200 \mu \mathrm{M}$ & $6 \pm 3^{\mathrm{a}}$ & $22 \pm 2^{\mathrm{a}}$ & $59 \pm 12^{\mathrm{a}}$ \\
\hline
\end{tabular}

Data are expressed as Mean \pm SEM for at least four separate experiments.

Isolated rat liver was perfused with different concentrations of drug and/or antioxidants and thiol reducing agents. NAC: N-acetylcysteine, DTT: Dithioteritol.

*Indicates significant increase in comparison with control (only liver) group $(p<0.05)$.

andicates significant decrease when compared with sulfasalazine-treated liver $(p<0.05)$.

Table 3. Effect of thiol-reductants and antioxidants on AST release from liver induced by sulfasalazine

\begin{tabular}{|c|c|c|c|}
\hline \multirow[b]{2}{*}{ Time (min): } & \multicolumn{3}{|c|}{ Perfusate AST level (U/L) } \\
\hline & 60 & 120 & 180 \\
\hline Control (only buffer) & $14 \pm 2$ & $34 \pm 4$ & $39 \pm 8$ \\
\hline+ Sulfasalazine $5 \mathrm{mM}$ & $38 \pm 10$ & $328 \pm 36^{*}$ & $2707 \pm 997^{*}$ \\
\hline + NAC $300 \mu \mathrm{M}$ & $30 \pm 11$ & $192 \pm 77^{\mathrm{a}}$ & $466 \pm 176^{\mathrm{a}}$ \\
\hline+ NAC $500 \mu \mathrm{M}$ & $34 \pm 4$ & $128 \pm 22^{\mathrm{a}}$ & $260 \pm 50^{\mathrm{a}}$ \\
\hline + DTT $200 \mu \mathrm{M}$ & $21 \pm 6$ & $335 \pm 133$ & $952 \pm 105^{\mathrm{a}}$ \\
\hline + DTT $400 \mu \mathrm{M}$ & $24 \pm 3$ & $74 \pm 27^{\mathrm{a}}$ & $303 \pm 143^{\mathrm{a}}$ \\
\hline+ Vitamin E $200 \mu \mathrm{M}$ & $18 \pm 4^{\mathrm{a}}$ & $61 \pm 14^{\mathrm{a}}$ & $197 \pm 46^{\mathrm{a}}$ \\
\hline+ Vitamin C $200 \mu \mathrm{M}$ & $12 \pm 1^{\mathrm{a}}$ & $37 \pm 2^{\mathrm{a}}$ & $175 \pm 70^{\mathrm{a}}$ \\
\hline
\end{tabular}

Data are shown as Mean \pm SEM for at least three separate experiments.

Isolated rat liver was perfused with different concentrations of drug and/or antioxidants and thiol reducing agents. NAC: N-acetylcysteine, DTT: Dithioteritol.

*Shows significant increase as compared with control (only buffer) group $(p<0.05)$.

andicates significant decrease when compared with sulfasalazine-treated liver $(p<0.05)$. 
Table 4. Effect of thiol-reductants and antioxidants on perfusate LDH level after sulfasalazine administration to isolated rat liver

\begin{tabular}{cccc}
\hline \hline & \multicolumn{3}{c}{ Perfusate LDH level (U/L) } \\
\hline Treatment & Time (min): & 60 & 120 \\
\hline Control (only buffer) & $10 \pm 3$ & $19 \pm 5$ & $26 \pm 4$ \\
+ Sulfasalazine 5 mM & $138 \pm 59^{*}$ & $387 \pm 114^{*}$ & $1146 \pm 223^{*}$ \\
+ NAC 300 $\mu \mathrm{M}$ & $14 \pm 14^{\mathrm{a}}$ & $296 \pm 23$ & $965 \pm 123$ \\
+ NAC 500 $\mu \mathrm{M}$ & $17 \pm 4^{\mathrm{a}}$ & $116 \pm 38^{\mathrm{a}}$ & $619 \pm 68^{\mathrm{a}}$ \\
+ DTT 200 $\mu \mathrm{M}$ & $30 \pm 8^{\mathrm{a}}$ & $793 \pm 266$ & $2080 \pm 185$ \\
+ DTT 400 $\mu \mathrm{M}$ & $4 \pm 1^{\mathrm{a}}$ & $258 \pm 57^{\mathrm{a}}$ & $161 \pm 50^{\mathrm{a}}$ \\
+ Vitamin E 200 $\mu \mathrm{M}$ & $17 \pm 2^{\mathrm{a}}$ & $156 \pm 80^{\mathrm{a}}$ & $821 \pm 402^{\mathrm{a}}$ \\
+ Vitamin C $200 \mu \mathrm{M}$ & $25 \pm 4^{\mathrm{a}}$ & $249 \pm 22^{\mathrm{a}}$ & $1386 \pm 120$ \\
\hline
\end{tabular}

Data are shown as Mean \pm SEM for at least three separate experiments.

Isolated rat liver was perfused with different concentrations of drug and/or antioxidants and thiol reducing agents. NAC: $\mathrm{N}$-acetylcysteine, DTT: Dithioteritol.

*Indicates significant increase as compared with control (only buffer) group $(p<0.05)$.

andicates significant decrease when compared with sulfasalazine-treated liver $(p<0.05)$.

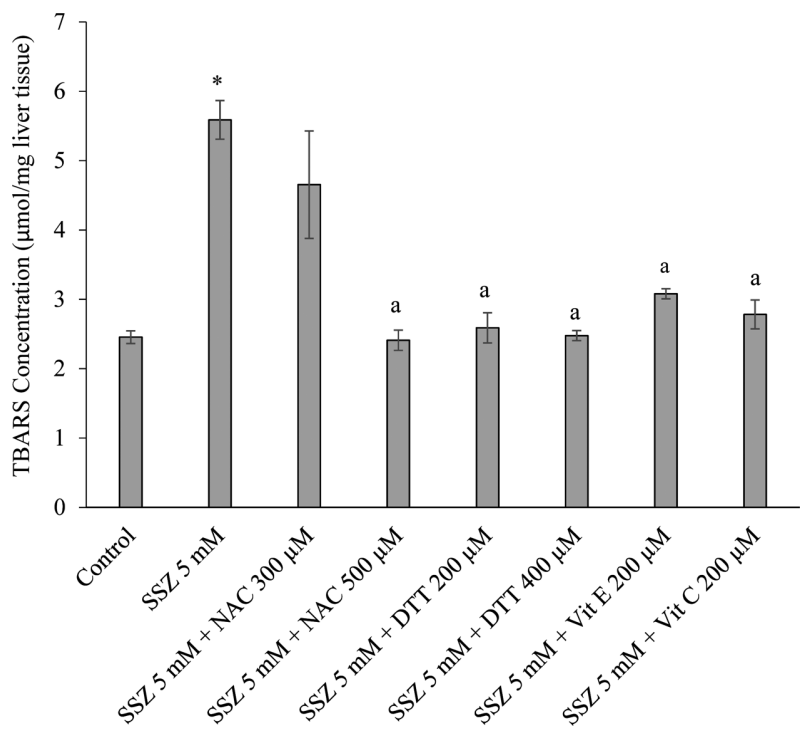

Fig. 1. Effects of antioxidants and thiol-reductants on lipid peroxidation induced by sulfasalazine in isolated rat liver. Data are represented as Mean \pm SEM for at least three independent experiments. SSZ: Sulfasalazine, NAC: N-acetylcysteine, DTT: Dithioteritol. *Indicates significantly higher than control group $(p<0.05)$. Indicates significantly lower when compared with sulfasalazine-treated group $(p<0.05)$.

It was found that a significant amount of thiobarbituric acid reactive substances (TBARS) were formed after sulfasalazine administration to the liver $(p<0.05)$ (Fig. 1). Moreover, a significant decrease in hepatic glutathione stores was detected in sulfasalazine-treated group $(p<0.05)$ (Fig. 2). Administration of antioxidants and thiol-reducing agents in the current investigation significantly reduced the level of lipid peroxidation in rat liver $(p<0.05)$ (Fig. 1). Furthermore, hepatic glutathione reservoirs maintained in a significantly higher level when sulfasalazine was co-administered with thiol-reducing agents and/or antioxidants $(p<0.05)$

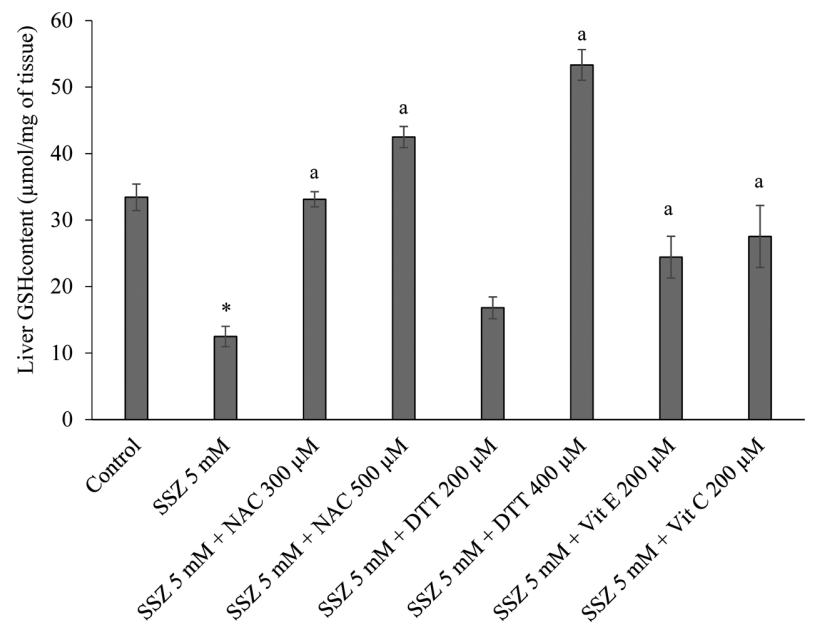

Fig. 2. Protective effects of antioxidants and thiol-reductants on hepatic glutathione depletion induced by sulfasalazine in isolated perfused rat liver. Data are given as Mean \pm SEM for at least three independent experiments. SSZ: Sulfasalazine, NAC: Nacetylcysteine, DTT: Dithioteritol. *Indicates significantly lower than control group $(p<0.05)$. Indicates significantly higher as compared to sulfasalazine-treated livers $(p<0.05)$.

\section{(Fig. 2).}

At the end of each experiment, livers were evaluated for histopathological lesions. It was found that sulfasalazine ( $5 \mathrm{mM})$ caused sinusoidal dilation, hepatocellular vacuolization, and tissue necrosis in comparison with control group (Fig. 3). Administration of NAC $(500 \mu \mathrm{M})$, DTT $(400 \mu \mathrm{M})$, vitamin $\mathrm{C}(200 \mu \mathrm{M})$ and vitamin $\mathrm{E}(200 \mu \mathrm{M})$, significantly alleviated liver histopathological changes induced by sulfasalazine (Fig. 3).

\section{DISCUSSION}

The objective of the present study was to investigate the 
Table 5. Graded histopathological changes of sulfasalazine-treated perfused rat liver

\begin{tabular}{lcccc}
\hline \hline Treatment & Hydropic changes & Vacuolization & Sinusoidal dilation & Necrosis \\
\hline Control & - & - & ++ & - \\
Sulfasalazine $5 \mathrm{mM}$ & - & +++ & +++ & ++ \\
Sulfasalazine $5 \mathrm{mM}$ + NAC $500 \mu \mathrm{M}$ & - & - & ++ & - \\
Sulfasalazine $5 \mathrm{mM}+$ DTT $400 \mu \mathrm{M}$ & - & + & ++ & - \\
Sulfasalazine $5 \mathrm{mM}$ + Vitamin E $400 \mu \mathrm{M}$ & - & ++ & ++ & - \\
Sulfasalazine $5 \mathrm{mM}$ + Vitamin C $400 \mu \mathrm{M}$ & - & ++ & ++ & - \\
\hline
\end{tabular}
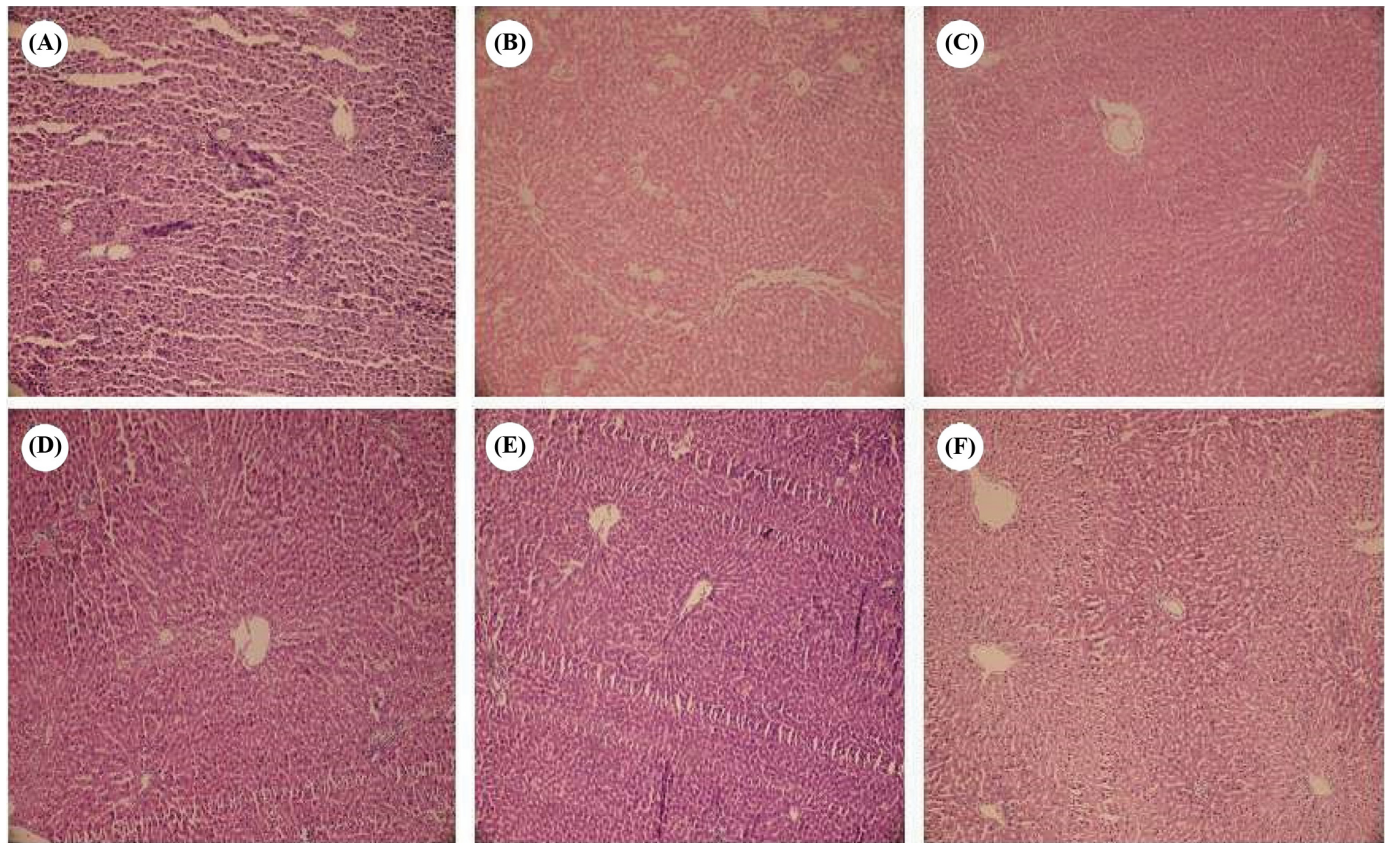

Fig. 3. Histopathological changes of perfused rat liver treated with sulfasalazine (H\&E staining). (A) control group perfused with Krebs-Henseleit buffer for 180 mins. No significant pathological changes except for a mild sinusoidal dilation was detected in this group. (B) Sulfasalazine $(5 \mathrm{mM})$ was perfused to isolated rat liver for 180 mins. Sinusoidal space dilation, hepatocellular vacuolization (Blue arrow) and cell necrosis (Yellow arrow) were observed in this group. (C) N-acetylcysteine (500 $\mu \mathrm{M})$ was co-administered with sulfasalazine for 180 mins. Only a mild sinusoidal space dilation was observed in this group. (D) Isolated rat liver was perfused by sulfasalazine $(5 \mathrm{mM})$ and dithioteritol $(400 \mu \mathrm{M})$ for 180 mins. A mild degree of hepatocellular vacuolization (Blue arrow) and sinusoidal dilation were pathological findings in this group. (E) Vitamin $C(200 \mu \mathrm{M})$ was administered with sulfasalazine $(5 \mathrm{mM})$ during $180 \mathrm{mins}$ of liver perfusion. (F) effect of vitamin $\mathrm{E}(200 \mu \mathrm{M})$ on sulfasalazine $(5 \mathrm{mM})$-induced histopathological lesions in perfused rat liver. Mild hepatocellular vacuolization and sinusoidal space dilation was found in groups (E) and (F).

role of thiol reducing agents and antioxidants against sulfasalazine-induced hepatic injury in an ex vivo model of isolated perfused rat liver. Sulfasalazine $(5 \mathrm{mM})$ caused hepatotoxicity as judged by a significant increase in perfusate biomarkers of liver injury, rise in tissue lipid peroxidation and depletion of liver glutathione reservoirs. Moreover, drug administration caused histopathological changes in the liver. The abovementioned markers of liver injury caused by sulfasalazine were alleviated by administration of thiolreducing agents and antioxidants.

Previous investigations proposed oxidative stress as a mechanism for sulfasalazine-induced liver injury $(12,14)$. In current study, we found that sulfasalazine caused a significant amount of lipid peroxidation as an index of oxidative biomembranes injury (Fig. 1). Moreover hepatic glutathione stores were decreased (Fig. 2), which might be a consequence of oxidative stress in the liver. The antioxidants and thiol-reducing agents effectively mitigated sulfasalazineinduced liver injury in our study. This might implicate the important role of thiol groups and oxidative stress in the organ injury caused by sulfasalazine. Thiol reductants and antioxidants might provide protection against sulfasalazine by counteracting oxidative stress in hepatocytes. Interestingly, it has been reported that NAC administration significantly alleviated severe sulfasalazine-induced reactions in human (22). This report might endorse the potential therapeutic capability of thiol-reductants against adverse events accompanied with sulfasalazine administration. It has been 
found that sulfasalazine blocks cysteine transport to glioma cells and consequently prevents glutathione synthesis (23), a mechanism which finally sensitize tumor cells to radio/ chemotherapy (23). Although such mechanism has not been proved in hepatocytes, it might be involved in sulfasalazineinduced liver injury and also in the potential protective effect of thiol reductants against sulfasalazine hepatotoxicity.

In large intestine, bacterial azoreductase enzymes cleave sulfasalazine to sulfapyridine and mesalazine. Sulfapyridine is almost completely absorbed compared with about $20 \sim 30 \%$ absorption for mesalazine. Approximately 10 30\% of the parent drug is also absorbed from the small intestine (24). In current investigation we aimed to reproduce sulfasalazine-induced hepatic injury in ex vivo model. Hence, we administered a higher concentration of sulfasalazine. This is actually different from in vivo conditions where only 10 $30 \%$ of intact sulfasalazine reaches the liver (24).

It is not clear that weather the whole molecule of sulfasalazine and/or its intestinal metabolites are responsible for the oxidative stress induction and finally the liver injury induced by this drug. Hence, further future investigations on the metabolic pathways of sulfasalazine and its intestinal metabolites might shed light on the mechanisms of liver injury induced by this drug.

Conditions in animal models and human subjects are highly different from in vitro/ex vivo experiments because of pharmacokinetic/pharmacodynamic parameters. However, results such as protective effects of thiol reducing agents and antioxidants against sulfasalazine-induced toxicity could help to clear the mechanisms of liver injury and may provide therapeutic strategies against drug hepatotoxicity. Furthermore, isolated organs are useful models for examining xenobiotics-induced injury without the complication of many interacting factors which are difficult to control in other experimental models. Hence, ex vivo models are effective tools for screening hepatoprotective compounds and studying the mechanisms of drug-induced liver injury. The protective agents in this model are potential subjects of

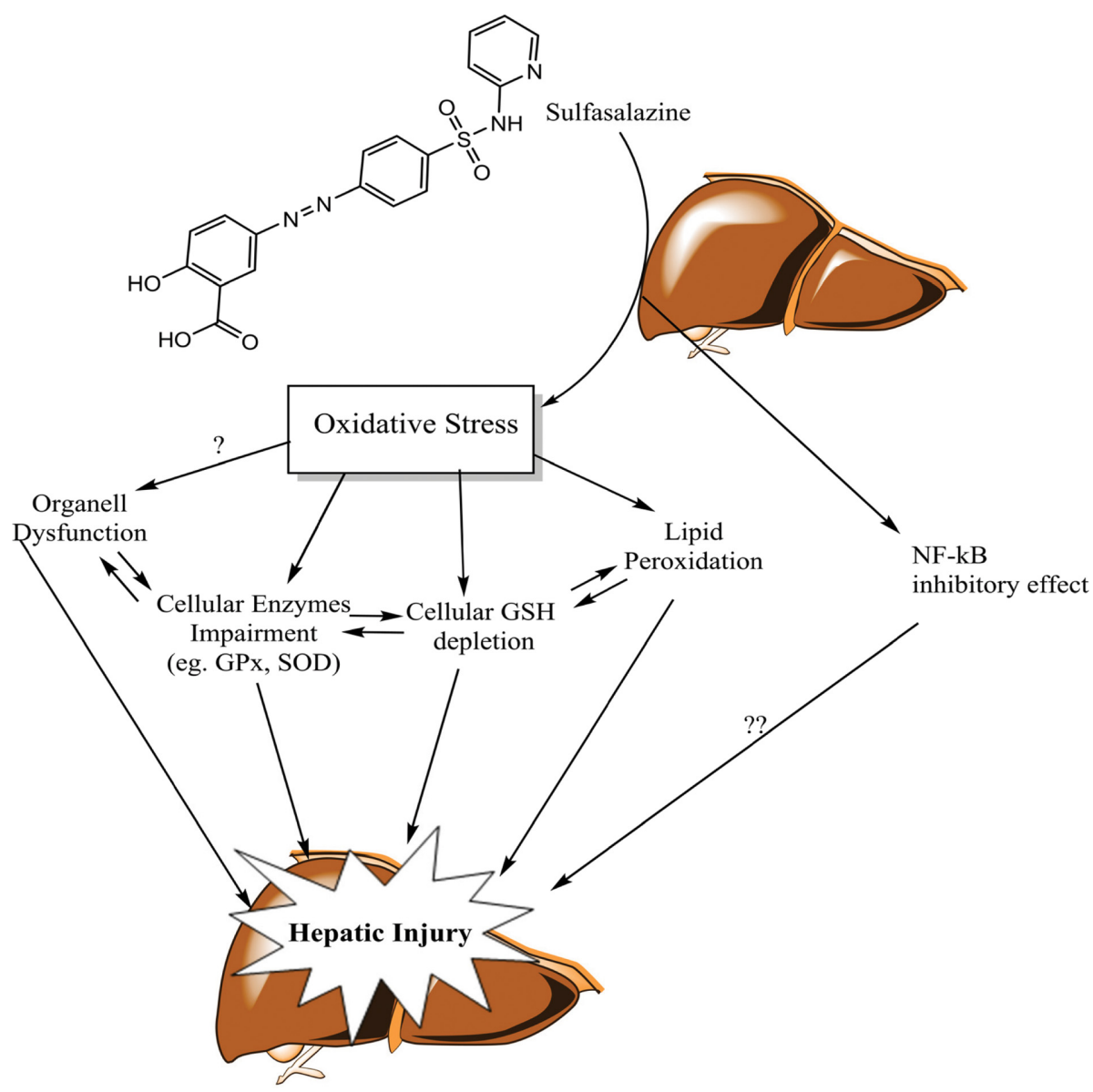

Fig. 4. The proposed mechanism(s) of liver injury induced by sulfasalazine and the hepatoprotection provided by thiol-reducing agents and antioxidants. Oxidative stress and its consequences such as biomembranes injury (lipid peroxidation) and glutathione reservoirs depletion are proposed to be involved in sulfasalazine-induced hepatotoxicity. Other complications such as cellular defense mechanisms impairment and organelle dysfunction might also be involved in sulfasalazine-induced liver injury. Antioxidants and thiol-reducing agents might provide hepatoprotection by counteracting sulfasalazine-induced oxidative stress in liver. 
further investigation in other models such as in vivo experiments.

The polymorphism in the $\mathrm{N}$-acetyl transferase (NAT) enzyme in different populations is postulated to be responsible in sulfasalazine adverse effects (25). However, the role of un-acetylated metabolites of sulfasalazine in the oxidative stress and the liver injury induced by this drug is not tested so far.

Some studies indicate the role of sulfasalazine in inhibiting nuclear factor kappa-B (NF- $\mathrm{KB})$ in different cells. It has been shown that sulfasalazine but not its metabolites, is a potent and specific inhibitor of NF- $\mathrm{KB}$ (26). This event might be related to the cytotoxicity of this chemical against cells such as lymphocytes (27), which might be related to the pharmacological effects of the drug (28). NF- $\kappa \mathrm{B}$ is involved in the activation of the anti-apoptotic genes transcription and cell proliferation (29). It is not clear weather inhibition of NF- $\kappa \mathrm{B}$ contribute in the liver injury induced by sulfasalazine (Fig. 4).

At least a part of sulfasalazine-induced toxic reactions including hepatic injury, seems to be immune-mediated (30). Due to the absence of immune system and its interactions with liver in current model, this investigation cannot predict the immune-mediated mechanisms of sulfasalazineinduced liver injury.

Although there is no clear consensus that they are effective, steroids have been used against sulfasalazine-induced liver injury in human cases (31). The data obtained from the current investigation suggest that antioxidants and thiolreducing agents could be a potential therapeutic choice against sulfasalazine-induced liver injury.

Altogether, the data obtained from current investigation indicate the role of oxidative stress in sulfasalazine-induced liver injury and the potential therapeutic properties of thiol reducing agents and antioxidants against this complication. The hepatoprotective properties of these agents deserve further investigation in other models of drug-induced liver injury. The proposed mechanisms of liver injury induced by sulfasalazine and the hepatoprotective role of antioxidants and thiol-reducing agents are summarized in Fig. 4.

\section{ACKNOWLEDGMENTS}

The authors thank Pharmaceutical Sciences Research Center (PSRC) of Shiraz University of Medical Sciences for providing instrumental facilities to carry out this study.

\section{REFERENCES}

1. Holt, M.P. and Ju, C. (2006) Mechanisms of drug-induced liver injury. AAPS J., 8, E48-E54.

2. Abdoli, N., Heidari, R., Azarmi, Y. and Eghbal, M.A. (2013) Mechanisms of the statins cytotoxicity in freshly isolated rat hepatocytes. J. Biochem. Mol. Toxicol., 27, 287-294.
3. Heidari, R., Niknahad, H., Jamshidzadeh, A. and Abdoli, N. (2014) Factors affecting drug-induced liver injury: antithyroid drugs as instances. Clin. Mol. Hepatol., 20, 237-248.

4. Heidari, R., Niknahad, H., Jamshidzadeh, A., Eghbal, M.A. and Abdoli, N. (2015) An overview on the proposed mechanisms of antithyroid drugs-induced liver injury. Adv. Pharm. Bull., 5, 1-11.

5. Rains, C.P., Noble, S. and Faulds, D. (1995) Sulfasalazine. A review of its pharmacological properties and therapeutic efficacy in the treatment of rheumatoid arthritis. Drugs, 50, 137156.

6. de Abajo, F.J., Montero, D., Madurga, M. and García Rodríguez, L.A. (2004) Acute and clinically relevant drug-induced liver injury: a population based case-control study. Br. J. Clin. Pharmacol., 58, 71-80.

7. Khokhar, O.S. and Lewis, J.H. (2010) Hepatotoxicity of agents used in the management of inflammatory bowel disease. Dig. Dis., 28, 508-518.

8. Marinos, G., Riley, J., Painter, D.M. and McCaughan, G.W. (1992) Sulfasalazine-induced fulminant hepatic failure. $J$. Clin. Gastroenterol., 14, 132-135.

9. Rubin, R. (1994) Sulfasalazine-induced fulminant hepatic failure and necrotizing pancreatitis. Am. J. Gastroenterol., 89, 789-91.

10. Ransford, R.A. and Langman, M.J. (2002) Sulphasalazine and mesalazine: serious adverse reactions re-evaluated on the basis of suspected adverse reaction reports to the committee on safety of medicines. Gut, 51, 536-539.

11. Jobanputra, P., Amarasena, R., Maggs, F., Homer, D., Bowman, S., Rankin, E., Filer, A., Raza, K. and Jubb, R. (2008) Hepatotoxicity associated with sulfasalazine in inflammatory arthritis: a case series from a local surveillance of serious adverse events. BMC Musculoskelet. Disord., 9, 48.

12. Linares, V., Alonso, V., Albina, M.L., Bellés, M., Sirvent, J.J., Domingo, J.L. and Sánchez, D.J. (2009) Lipid peroxidation and antioxidant status in kidney and liver of rats treated with sulfasalazine. Toxicology, 256, 152-156.

13. Linares, V., Alonso, V. and Domingo, J.L. (2011) Oxidative stress as a mechanism underlying sulfasalazine-induced toxicity. Expert Opin. Drug Saf., 10, 253-263.

14. Alonso, V., Linares, V., Bellés, M., Albina, M.L., Sirvent, J.J., Domingo, J.L. and Sánchez, D.J. (2009) Sulfasalazine induced oxidative stress: A possible mechanism of male infertility. Reprod. Toxicol., 27, 35-40.

15. Wolkoff, A.W., Johansen, K.L. and Goeser, T. (1987) The isolated perfused rat liver: preparation and application. Anal. Biochem., 167, 1-14.

16. Ferrigno, A., Richelmi, P. and Vairetti, M. (2013) Troubleshooting and improving the mouse and rat isolated perfused liver preparation. J. Pharmacol. Toxicol. Methods, 67, 107114.

17. Bessems, M., 't Hart, N.A., Tolba, R., Doorschodt, B.M., Leuvenink, H.G., Ploeg, R.J., Minor, T. and van Gulik, T.M. (2006) The isolated perfused rat liver: standardization of a time-honoured model. Lab. Anim., 40, 236-246.

18. Heidari, R., Jamshidzadeh, A., Keshavarz, N. and Azarpira, N. (2015) Mitigation of methimazole-induced hepatic injury by taurine in mice. Sci. Pharm., 83, 143-158.

19. Heidari, R., Babaei, H. and Eghbal, M.A. (2013) cytoprotec- 
tive effects of organosulfur compounds against methimazoleinduced toxicity in isolated rat hepatocytes. Adv. Pharm. Bull., 3, 135-142.

20. Sedlak, J. and Lindsay, R.H. (1968) estimation of total, protein-bound, and nonprotein sulfhydryl groups in tissue with Ellman's reagent. Anal. Biochem., 25, 192-205.

21. Heidari, R., Babaei, H., Roshangar, L. and Eghbal, M.A. (2014) Effects of enzyme induction and/or glutathione depletion on methimazole-induced hepatotoxicity in mice and the protective role of N-acetylcysteine. Adv. Pharm. Bull., 4, 2128.

22. Gabay, C., De Bandt, M. and Palazzo, E. (1993) Sulphasalazine-related life-threatening side effects: is $\mathrm{N}$-acetylcysteine of therapeutic value? Clin. Exp. Rheumatol., 11, 417-20.

23. Sleire, L., Skeie, B.S., Netland, I.A., Førde, H.E., Dodoo, E., Selheim, F., Leiss, L., Heggdal, J.I., Pedersen, P.H., Wang, J. and Enger, P.Ø. (2015) Drug repurposing: sulfasalazine sensitizes gliomas to gamma knife radiosurgery by blocking cystine uptake through system $\mathrm{xc}^{-}$, leading to glutathione depletion. Oncogene, 34, 5951-5959.

24. Plosker, G.L. and Croom, K.F. (2005) Sulfasalazine: a review of its use in the management of rheumatoid arthritis. Drugs, 65, 1825-1849.
25. Makarova, S.I. (2008) Human N-acetyltransferases and druginduced hepatotoxicity. Curr. Drug Metab., 9, 538-545.

26. Wahl, C., Liptay, S., Adler, G. and Schmid, R.M. (1998) Sulfasalazine: a potent and specific inhibitor of nuclear factor kappa B. J. Clin. Invest., 101, 1163-1174.

27. Liptay, S., Bachem, M., Häcker, G., Adler, G., Debatin, K.M. and Schmid, R.M. (1999) Inhibition of nuclear factor kappa B and induction of apoptosis in T-lymphocytes by sulfasalazine. Br. J. Pharmacol., 128, 1361-1369.

28. Yamamoto, Y. and Gaynor, R.B. (2001) Therapeutic potential of inhibition of the NF-kB pathway in the treatment of inflammation and cancer. J. Clin. Invest., 107, 135-142.

29. Karin, M. and Lin, A. (2002) NF- $\mathrm{KB}$ at the crossroads of life and death. Nat. Immunol., 3, 221-227.

30. Mihas, A.A., Goldenberg, D.J. and Slaughter, R.L. (1978) Sulfasalazine toxic reactions: hepatitis, fever, and skin rash with hypocomplementemia and immune complexes. JAMA, 239, 2590-2591.

31. Boyer, D.L., Li, B.U., Fyda, J.N. and Friedman, R.A. (1989) Sulfasalazine-induced hepatotoxicity in children with inflammatory bowel disease. J. Pediatr. Gastroenterol. Nutr., 8, 528532. 